\title{
Analogues of Kahan's method for higher order equations of higher degree
}

\author{
A.N.W. Hone ${ }^{\star}$ and G.R.W. Quispel \\ 1 School of Mathematics, Statistics \& Actuarial Science, University of Kent, \\ Canterbury CT2 7NF, UK, A.N.W.Hone@kent.ac.uk \\ 2 Department of Mathematics and Statistics, La Trobe University, Bundoora, \\ Victoria 3086, Australia, R.Quispel@latrobe.edu.au
}

\begin{abstract}
Kahan introduced an explicit method of discretization for systems of first order differential equations with nonlinearities of degree at most two (quadratic vector fields). Kahan's method has attracted much interest due to the fact that it preserves many of the geometrical properties of the original continuous system. In particular, a large number of Hamiltonian systems of quadratic vector fields are known for which their Kahan discretization is a discrete integrable system. In this note, we introduce a special class of explicit order-preserving discretization schemes that are appropriate for certain systems of ordinary differential equations of higher order and higher degree.
\end{abstract}

\section{Introduction}

Kahan's method is a special discretization scheme that provides an explicit method for integrating quadratic vector fields, given by systems of first order ordinary differential equations (ODEs) of the form

$$
\frac{\mathrm{d} x_{i}}{\mathrm{~d} t}=f_{i}\left(x_{1}, \ldots, x_{N}\right), \quad i=1, \ldots, N,
$$

where each function $f_{i}$ is a polynomial of total degree two in the independent variables $x_{1}, \ldots, x_{N}$ (see [15] or [16]). In order to specify Kahan's method, one should replace each derivative on the left-hand side of (1) by the forward difference, so that

$$
\frac{\mathrm{d} x_{i}}{\mathrm{~d} t} \rightarrow \Delta x_{i}:=\frac{\bar{x}_{i}-x_{i}}{h},
$$

while terms of degrees two, one and zero appearing in each $f_{i}$ on the right-hand side are replaced according to the rules

$$
x_{j} x_{k} \rightarrow \frac{1}{2}\left(\bar{x}_{j} x_{k}+x_{j} \bar{x}_{k}\right), \quad x_{j} \rightarrow \frac{1}{2}\left(x_{j}+\bar{x}_{j}\right), \quad c \rightarrow c,
$$

where $h$ is the time step and $\bar{x}_{i}$ denotes the approximation to $x_{i}(t+h)$.

\footnotetext{
* Work begun on leave at School of Mathematics \& Statistics, University of New South Wales, NSW 2052, Australia.
} 
It was noticed some time ago that Kahan's method provides an effective integration scheme for the classic two-species Lotka-Volterra model

$$
\frac{\mathrm{d} x}{\mathrm{~d} t}=\alpha x(1-y), \quad \frac{\mathrm{d} y}{\mathrm{~d} t}=y(x-1)
$$

(with $\alpha>0$ being an arbitrary parameter), retaining the qualitative features of the orbits of the continuous system, namely the stability of orbits around the elliptic fixed point at $(x, y)=(1,1)$. This was subsequently explained by the fact that the Kahan discretization of (3), given by

$$
\begin{aligned}
(\bar{x}-x) / h & =\frac{\alpha}{2}(x(1-\bar{y})+\bar{x}(1-y)), \\
(\bar{y}-y) / h & =\frac{1}{2}(y(\bar{x}-1)+\bar{y}(x-1)),
\end{aligned}
$$

preserves the same symplectic form

$$
\omega=\frac{\mathrm{d} x \wedge \mathrm{d} y}{x y}
$$

as the original Hamiltonian system [26]. In the context of Lotka-Volterra models, a variant of Kahan's method with similar properties was discovered by Mickens [19], who had previously considered various examples of nonstandard discretization methods [18], but a more rapid growth of interest in Kahan's method began when Hirota and Kimura independently proposed the rules (2) for the discretization of the Euler equations for rigid body motion, finding that the resulting discrete system is also completely integrable [11], and this has led to the search for other discrete integrable systems arising in this way [12], with a survey of several results given in [20], and some more recent examples in [21] and [22], for instance.

Many of the geometrical properties of Kahan's method for quadratic vector fields are based on the polarization identity for quadratic forms [3], and recently this has led to a generalization of Kahan's method that can cope with vector fields of degree three or more, by using higher degree analogues of polarization [4]. One disadvantage of the latter method for higher degree vector fields is that, in common with multistep methods in numerical analysis, one must use extra grid points for the discretization, so the original ODE system does not provide enough initial values to start the iteration of the discrete version. However, if one is looking for a discretization scheme that preserves integrability or other geometric properties of ODEs, then it is desirable for the initial value space of the discrete system to have the same dimension as that of the continuous one. Here we would like to suggest a discretization scheme with the latter property, which is a natural generalization of Kahan's method to higher order and higher degree.

The idea is to consider a system of ODEs of order $n \geq 1$, with the right-hand sides being functions of the coordinates $x_{1}, \ldots, x_{N}$ only, of the form

$$
\frac{\mathrm{d}^{n} x_{i}}{\mathrm{~d} t^{n}}=f_{i}\left(x_{1}, \ldots, x_{N}\right), \quad i=1, \ldots, N,
$$


where each function $f_{i}$ is a polynomial of maximal degree $n+1$. For $n=1$ this is a quadratic vector field, which one can discretize using Kahan's method. In the next section, we present an explicit discretization scheme for systems of the form (4), valid for any $n \geq 1$, which reduces to Kahan's method when $n=1$. The first new case is $n=2$, corresponding to systems of Newton equations, which are relevant in many applications. We illustrate this in section 3 by considering the discretization of the motion of a single particle moving in a quartic potential. The latter is one of the simplest examples of an integrable Hamiltonian system, and it turns out that the discrete version produced by the method is also integrable, with a conserved quantity and an invariant symplectic form. In section 4, we consider a different example of fourth order, namely a nonlinear beam equation, and briefly compare the discretization obtained by the new method with another discretization obtained by applying an approach similar to Kahan's directly to the Lagrangian of the continuous system.

\section{A higher order version of Kahan's method}

For $n=2$, (4) becomes a system of Newton equations, assumed to have polynomial forces of degree at most three, which can be conveniently written as

$$
\frac{\mathrm{d}^{2} x_{i}}{\mathrm{~d} t^{2}}=\sum_{0 \leq j_{1} \leq j_{2} \leq j_{3} \leq N} c_{i, j_{1}, j_{2}, j_{3}} x_{j_{1}} x_{j_{2}} x_{j_{3}}, \quad i=1, \ldots, N,
$$

where $c_{i j k \ell}$ are arbitrary coefficients, and we have included an additional dummy variable $x_{0}=1$ to allow terms of degree less than three to be included within the same summation. Then to discretize (5) we propose the following:

$$
\frac{\bar{x}_{i}-2 x_{i}+\underline{x}_{i}}{h^{2}}=\frac{1}{6} \sum_{\sigma \in S_{3}} \sum_{0 \leq j_{1} \leq j_{2} \leq j_{3} \leq N} c_{i, j_{1}, j_{2}, j_{3}} \underline{x}_{j_{\sigma(1)}} x_{j_{\sigma(2)}} \bar{x}_{j_{\sigma(3)}},
$$

for $i=1, \ldots, N$; the first summation is over permutations $\sigma$ in $S_{3}$, the symmetric group on three symbols, and $\bar{x}_{i}=x^{(1)}, \underline{x}_{i}=x^{(-1)}$ are the approximations to $x_{i}(t \pm h)$, with time step $h$. For terms of degree three, with each variable $x_{j}$ appearing at the three adjacent lattice points $\underline{x}_{j}=x_{j}^{(-1)}, x_{j}=x_{j}^{(0)}, \bar{x}_{j}=x_{j}^{(1)}$, the replacement rule is described explicitly by

$$
x_{j} x_{k} x_{\ell} \rightarrow \frac{1}{6}\left(\underline{x}_{j} x_{k} \bar{x}_{\ell}+\underline{x}_{j} \bar{x}_{k} x_{\ell}+x_{j} \underline{x}_{k} \bar{x}_{\ell}+x_{j} \bar{x}_{k} \underline{x}_{\ell}+\bar{x}_{j} x_{k} \underline{x}_{\ell}+\bar{x}_{j} \underline{x}_{k} x_{\ell}\right),
$$

while for terms of degree two the rule is obtained by setting $\ell=0$, so that $x_{\ell} \rightarrow x_{0}=1$ in the above, and for the linear terms one can set $k=\ell=0$, so that the rule for terms of degree less than three is

$$
\begin{aligned}
x_{j} x_{k} & \rightarrow \frac{1}{6}\left(\underline{x}_{j} x_{k}+\underline{x}_{j} \bar{x}_{k}+x_{j} \underline{x}_{k}+x_{j} \bar{x}_{k}+\bar{x}_{j} x_{k}+\bar{x}_{j} \underline{x}_{k}\right), \\
x_{j} & \rightarrow \frac{1}{3}\left(\underline{x}_{j}+x_{j}+\bar{x}_{j}\right), \quad c \rightarrow c .
\end{aligned}
$$


Following the approach of [4], a second order system of equations can be written in vector form as

$$
\frac{\mathrm{d}^{2} \mathbf{x}}{\mathrm{d} t^{2}}=\mathbf{f}(\mathbf{x})
$$

where each component of the vector of functions $\mathbf{f}=\left(f_{1}, f_{2}, \ldots, f_{N}\right)^{T}$ is a polynomial of degree at most three, and then the replacement rules (7) and (8) are equivalent to the formula

$$
\begin{aligned}
\frac{1}{h^{2}}(\overline{\mathbf{x}}-2 \mathbf{x}+\underline{\mathbf{x}})= & \frac{9}{2} \mathbf{f}\left(\frac{\overline{\mathbf{x}}+\mathbf{x}+\underline{\mathbf{x}}}{3}\right)-\frac{4}{3}\left(\mathbf{f}\left(\frac{\underline{\mathbf{x}}+\mathbf{x}}{2}\right)+\mathbf{f}\left(\frac{\underline{\mathbf{x}}+\overline{\mathbf{x}}}{2}\right)+\mathbf{f}\left(\frac{\mathbf{x}+\overline{\mathbf{x}}}{2}\right)\right) \\
& +\frac{1}{6}(\mathbf{f}(\underline{\mathbf{x}})+\mathbf{f}(\mathbf{x})+\mathbf{f}(\overline{\mathbf{x}})) .
\end{aligned}
$$

Proposition 1. The discretization (10) commutes with affine transformations

$$
\mathbf{y} \mapsto \mathbf{x}=A \mathbf{y}+\mathbf{b},
$$

where $A \in G L(N, \mathbb{R})$ is a constant matrix and $\mathbf{b} \in \mathbb{R}^{N}$ is a vector of constants.

Proof. Under the transformation (11), f $(\cdot)$ in (9) is replaced by $A^{-1} \mathbf{f}(A \cdot+\mathbf{b})$. Upon substituting (11) and its shifted versions into (10), it is not hard to check that the same occurs for each appearance of $\mathbf{f}$ on the right-hand side.

The symmetric replacement rules above generalize to any order $n \geq 1$, so that for a system of $n$th order ODEs (4) with right-hand sides all of degree $n+1$ the discretization becomes

$$
\Delta^{n} x_{i}=\frac{1}{(n+1) !} \sum_{\sigma \in S_{n+1}} \sum_{j_{k} \leq j_{k+1}} c_{i, j_{1}, \ldots, j_{n+1}} x_{j_{\sigma(1)}} x_{j_{\sigma(2)}}^{(1)} x_{j_{\sigma(3)}}^{(2)} \cdots x_{j_{\sigma(n+1)}}^{(n)},
$$

for $i=1, \ldots, N$, with $x_{i}^{(1)}=\bar{x}_{i}, x_{i}^{(2)}=\overline{\bar{x}}_{i}, \ldots, x_{j}^{(n)}$ corresponding to shifts by steps of $h, 2 h, \ldots, n h$, and the interior summation being for $0 \leq j_{1} \leq \cdots \leq$ $j_{n+1} \leq N$. On the left-hand side of (12) we have replaced the $n$th derivative by the $n$th power of the forward difference operator, and for convenience we have written everything on the right-hand side in terms of forward shifts of the variables $x_{j}$. The discretization (12) reduces to Kahan's method when $n=1$, and to (6) when $n=2$, modulo shifting the lattice points $-1,0,1$ in the latter up to $0,1,2$.

Clearly there are other choices of discrete $n$th derivative that one could take, and other affine combinations of terms with the same homogeneous degree could be chosen while preserving the continuum limit. We have taken the most symmetrical choice in (12), because it is manifestly linear in each of the highest shifts $x_{1}^{(n)}, x_{2}^{(n)} \ldots, x_{N}^{(n)}$, so it can be explicitly solved for each of these quantities to yield rational functions of all the lower shifts. It is also linear in each of the lowest shifts $x_{i}=x_{i}^{(0)}$ for $i=1, \ldots, N$, so it can be explicitly solved for these as well. Thus (12) is an implicit way of writing an explicit birational map in dimension $n N$, corresponding to $\mathbf{X} \mapsto \overline{\mathbf{X}}$, where

$$
\begin{aligned}
& \mathbf{X}=\left(x_{1}^{(0)}, \ldots, x_{N}^{(0)}, x_{1}^{(1)}, \ldots, x_{N}^{(1)}, \ldots, x_{1}^{(n-1)}, \ldots, x_{N}^{(n-1)}\right), \\
& \overline{\mathbf{X}}=\left(x_{1}^{(1)}, \ldots, x_{N}^{(1)}, x_{1}^{(2)}, \ldots, x_{N}^{(2)}, \ldots, x_{1}^{(n)}, \ldots, x_{N}^{(n)}\right) .
\end{aligned}
$$




\section{Discretization of a quartic oscillator}

To see why it might be worth investigating these higher Kahan-like schemes, we start by presenting the following example: $n=2$ with a cubic force on a particle in one dimension, generated by a natural Hamiltonian with a quartic potential, that is

$$
H=\frac{1}{2} p^{2}+\frac{1}{4} a x^{4}+\frac{1}{3} b x^{3}+\frac{1}{2} c x^{2}+d x,
$$

which yields the Newton equation

$$
\ddot{x}=-a x^{3}-b x^{2}-c x-d .
$$

This is an integrable system par excellence, and the generic level sets $H=$ const are quartic curves of genus one in the $(x, p)$ plane. The discretization (6) applied to (13) produces a difference equation of second order, given by

$$
\bar{x}=\frac{(3-\gamma) x-\delta-(\beta x+\gamma) \underline{x}}{\beta x+\gamma+(\alpha x+\beta) \underline{x}},
$$

where

$$
\alpha=a h^{2}, \quad \beta=\frac{b h^{2}}{3}, \quad \gamma=1+\frac{c h^{2}}{3}, \quad \delta=d h^{2} .
$$

The map (14) is an example of a QRT map [25], but let us suppose that we do not know the geometric properties of this map. To find these properties, such as the existence of a preserved measure, and first and second integrals of the map (14), we will look for preserved Darboux polynomials, as detailed in our recent work [5] and [6]. To this end, we write the second order equation (14) as two first order ones, namely

$$
\bar{x}=y, \quad \bar{y}=\frac{(3-\gamma) y-\delta-(\beta y+\gamma) x}{\beta y+\gamma+(\alpha y+\beta) x},
$$

and look for polynomials $P$ satisfying

$$
P(\bar{x}, \bar{y})=J(x, y) P(x, y)
$$

where $J$ is the Jacobian determinant of the map (15), i.e.

$$
J(x, y)=\frac{(\beta y+\gamma)^{2}+(\alpha y+\beta)((3-\gamma) y-\delta)}{(\alpha x y+\beta(x+y)+\gamma)^{2}}
$$

Substituting (17) into (16), and looking for polynomials up to total degree four in $x$ and $y$, we find two linearly independent solutions, given by

$$
P_{1}=\alpha x y+\beta(x+y)+\gamma,
$$

$P_{2}=\left(\alpha \gamma-\beta^{2}\right) x^{2} y^{2}+\epsilon x y(x+y)+\zeta\left(x^{2}+y^{2}\right)-(3-\gamma)^{2} x y+(3-\gamma) \delta(x+y)-\delta^{2}$, 
with

$$
\epsilon=\alpha \delta+\beta(3-\gamma), \quad \zeta=\beta \delta+\gamma(3-\gamma) .
$$

It follows that the map (14) is measure-preserving, with the invariant symplectic form

$$
\frac{\mathrm{d} x \wedge \mathrm{d} y}{P_{1}}=\frac{\mathrm{d} x \wedge \mathrm{d} y}{\alpha x y+\beta(x+y)+\gamma},
$$

and the first integral

$$
I=\frac{P_{2}}{P_{1}}
$$

given by

$$
\frac{\left(\alpha \gamma-\beta^{2}\right) x^{2} y^{2}+\epsilon x y(x+y)+\zeta\left(x^{2}+y^{2}\right)-(3-\gamma)^{2} x y+(3-\gamma) \delta(x+y)-\delta^{2}}{\alpha x y+\beta(x+y)+\gamma} .
$$

Hence the integrability is preserved by the discretization in this case, and we recover the standard property of a QRT map, that it preserves a pencil of biquadratic curves, here given by

$$
\lambda P_{1}(x, y)+P_{2}(x, y)=0 .
$$

Moreover, in the continuum limit $h \rightarrow 0$, the standard area form $\mathrm{d} x \wedge \mathrm{d} y$ and the Hamiltonian $H$ are recovered from (18) and (19) respectively, since from $y=x+h p+O\left(h^{2}\right)$ we find

$$
P_{1}=1+O\left(h^{2}\right), \quad P_{2}=4 H h^{2}+O\left(h^{3}\right) .
$$

The equation (13) includes Duffing's equation, which is the case $b=d=0$, and also the second order ODE for the Weierstrass $\wp$ function, which arises when $a=c=0$. In [23], another replacement rule is used for the cubic and linear terms in Duffing's equation, somewhat less symmetrical than the one defined by (7), and it is shown that if the coefficients and denominator in the second difference operator are replaced by suitable functions of the parameters and the time step $h$ then this alternative rule results in a discretization that is exact, in the sense that the iterates of the difference equation interpolate the solution of the original ODE. Similarly, in [24] an exact discretization is obtained for the case corresponding to the Weierstrass $\wp$ function, with only quadratic and constant terms on the right-hand side. However, in the latter case, the exact discretization (derived from the addition formula for the $\wp$ function) requires not only a different replacement rule for the quadratic terms compared with (8), but also extra cubic and linear terms that must be included, with a coefficient which is $O\left(h^{2}\right)$. When $a=c=0$, the equation (13) can be rewritten as a quadratic vector field, namely

$$
\frac{\mathrm{d} x}{\mathrm{~d} t}=p, \quad \frac{\mathrm{d} p}{\mathrm{~d} t}=-b x^{2}-d,
$$


so that Kahan's method can be applied, as in [20], resulting in a first order discrete system which is equivalent to a second order difference equation for $x$, namely

$$
\bar{x}+\underline{x}=\frac{4 x-2 \delta}{3 \beta x+2}
$$

(where we set $\beta=b h^{2} / 3, \delta=d h^{2}$ as before). The equation (20) is a QRT map in additive form, clearly of a different type to (14), which becomes

$$
\bar{x}=\frac{2 x-\delta-(\beta x+1) \underline{x}}{\beta(x+\underline{x})+1}
$$

when $\alpha=0, \gamma=1$. To see that they are really different QRT maps, in the sense that they are not related to one another via so-called curve-dependent McMillan maps [14], observe that the pencil of invariant biquadraic curves corresponding to $(20)$ is

$$
\lambda-\beta^{2} x^{2} y^{2}+\frac{4}{3} \beta x y(x+y)+\frac{4}{3}\left(x^{2}+y^{2}\right)-\frac{2}{3}(4+\beta \delta) x y+\frac{4}{3} \delta(x+y)=0,
$$

whereas when $\alpha=0, \gamma=1$ the pencil $\lambda P_{1}(x, y)+P_{2}(x, y)=0$ for (14) reduces to one of a different type, namely

$\lambda(1+\beta(x+y))-\beta^{2} x^{2} y^{2}+2 \beta x y(x+y)+(\beta \delta+2)\left(x^{2}+y^{2}\right)-4 x y+2 \delta(x+y)-\delta^{2}=0$.

\section{Two discretizations of a nonlinear beam equation}

Vibrating beams were considered by Leonardo da Vinci [7], but the traditional theory of vibrations of a beam is usually attributed to Euler and Bernoulli [10], being described by a partial differential equation (PDE) of fourth order, which in dimensionless form is given by

$$
\frac{\partial^{2} w}{\partial t^{2}}+\frac{\partial^{4} w}{\partial x^{4}}=Q .
$$

For the case of a static beam, the equation has the form

$$
\frac{\mathrm{d}^{4} w}{\mathrm{~d} x^{4}}=Q
$$

where $w=w(x)$ is the vertical deflection of the beam, which lies horizontally along the $x$-axis. The standard beam model is linear, with the distributed load $Q$ on the right-hand size being a constant (or more generally, a function of $x$, the independent variable). However, here we consider a more general nonlinear version of the model, derived from a second order Lagrangian of the form

$$
L=\frac{1}{2}\left(\frac{\mathrm{d}^{2} w}{\mathrm{~d} x^{2}}\right)^{2}-V(w)
$$


which gives a nonlinear load function

$$
Q(w)=\frac{\mathrm{d} V}{\mathrm{~d} w} .
$$

In the linear case, the model was considered recently from the viewpoint of a Hamilton-Jacobi approach to higher order implicit systems [8], while a coupled PDE system of beam equations with cubic nonlinearity was analysed in [27]. From the second order Lagrangian (21), we can introduce the Ostrogradsky variables (see [1], for instance), given by

$$
q_{1}=w, \quad q_{2}=w^{\prime}, \quad p_{1}=\frac{\partial L}{\partial w^{\prime}}-\frac{\mathrm{d}}{\mathrm{d} x}\left(\frac{\partial L}{\partial w^{\prime \prime}}\right)=-w^{\prime \prime \prime}, \quad p_{2}=\frac{\partial L}{\partial w^{\prime \prime}}=w^{\prime \prime},
$$

where the primes denote derivatives with respect to the independent variable $x$. Then $\left(q_{1}, p_{1}\right),\left(q_{2}, p_{2}\right)$ provide two pairs of canonically conjugate positions and momenta, and the Euler-Lagrange equation

$$
\frac{\mathrm{d}^{2}}{\mathrm{~d} x^{2}}\left(\frac{\partial L}{\partial w^{\prime \prime}}\right)-\frac{\mathrm{d}}{\mathrm{d} x}\left(\frac{\partial L}{\partial w^{\prime}}\right)+\frac{\partial L}{\partial w}=0
$$

which for the Lagrangian (22) is given by (21) with $Q=\mathrm{d} V / \mathrm{d} w$, is equivalent to Hamilton's equations for the Hamiltonian function

$$
H=\frac{1}{2}\left(p_{2}\right)^{2}+q_{2} p_{1}+V\left(q_{1}\right) .
$$

For the sake of concreteness, we consider the case of an odd potential

$$
V(w)=\frac{a}{5} w^{5}+\frac{b}{3} w^{3}+c w,
$$

so that the nonlinear beam equation is given by

$$
w^{\prime \prime \prime \prime}=a w^{4}+b w^{2}+c .
$$

To begin with, we consider the result of applying the discretization rule (12) to the nonlinear beam equation (24), which produces a difference equation of fourth order, of the form

$$
\Delta^{4} w=F\left(w^{(0)}, w^{(1)}, w^{(2)}, w^{(3)}, w^{(4)}\right)
$$

for a function $F$ that is a sum of terms of total degree four, two and zero. This can be written more symmetrically by shifting down by two steps, to yield

$$
\frac{w^{(-2)}-4 w^{(-1)}+6 w^{(0)}-4 w^{(1)}+w^{(2)}}{h^{4}}=F_{4}+F_{2}+c,
$$

where the quartic terms are specified by

$$
\begin{aligned}
\frac{5}{a} F_{4}= & w^{(-2)} w^{(-1)} w^{(0)} w^{(1)}+w^{(-2)} w^{(-1)} w^{(0)} w^{(2)}+w^{(-2)} w^{(-1)} w^{(1)} w^{(2)} \\
& +w^{(-2)} w^{(0)} w^{(1)} w^{(2)}+w^{(-1)} w^{(0)} w^{(1)} w^{(2)},
\end{aligned}
$$


and the quadratic terms are given by

$$
\begin{aligned}
\frac{10}{b} F_{2}= & w^{(-2)} w^{(-1)}+w^{(-2)} w^{(0)}+w^{(-2)} w^{(1)}+w^{(-2)} w^{(2)}+w^{(-1)} w^{(0)} \\
& +w^{(-1)} w^{(1)}+w^{(-1)} w^{(2)}+w^{(0)} w^{(1)}+w^{(0)} w^{(2)}+w^{(1)} w^{(2)} .
\end{aligned}
$$

It turns out that the birational map defined by (26) is measure-preserving. This is a consequence of the fact that the formula for the right-hand side of (25) is both linear and symmetric in its arguments, so that the derivatives with respect to the highest and lowest shifts, namely

$$
\frac{\partial F}{\partial w^{(0)}}=G\left(w^{(1)}, w^{(2)}, w^{(3)}, w^{(4)}\right), \quad \frac{\partial F}{\partial w^{(4)}}=H\left(w^{(0)}, w^{(1)}, w^{(2)}, w^{(3)}\right),
$$

are very closely related to one another.

Proposition 2. The discretization (26) preserves the volume form

$$
\Omega=\frac{1}{1-h^{2} H\left(w^{(-2)}, w^{(-1)}, w^{(0)}, w^{(1)}\right)} \mathrm{d} w^{(-2)} \wedge \mathrm{d} w^{(-1)} \wedge \mathrm{d} w^{(0)} \wedge \mathrm{d} w^{(1)},
$$

where $H$ is defined by (29).

Proof. Upon taking the differential of both sides of (26), we obtain the equation

$$
\begin{aligned}
& \left(1-h^{2} G\left(w^{(-1)}, w^{(0)}, w^{(1)}, w^{(2)}\right)\right) \mathrm{d} w^{(-2)} \\
& +\left(1-h^{2} H\left(w^{(-2)}, w^{(-1)}, w^{(0)}, w^{(1)}\right)\right) \mathrm{d} w^{(2)}+\cdots=0,
\end{aligned}
$$

where the ellipsis denotes terms that are linear in $\mathrm{d} w^{(-1)}, \mathrm{d} w^{(0)}$ and $\mathrm{d} w^{(1)}$. The result then follows from taking the wedge product of the equation above with $\mathrm{d} w^{(-1)} \wedge \mathrm{d} w^{(0)} \wedge \mathrm{d} w^{(1)}$, and noting the identity

$$
G\left(w^{(-1)}, w^{(0)}, w^{(1)}, w^{(2)}\right)=H\left(w^{(-1)}, w^{(0)}, w^{(1)}, w^{(2)}\right),
$$

which follows from the symmetry of $F$.

When $a, b$ are not both zero, so that the nonlinear terms are present, the above discretization of (24) cannot be obtained from a second order discrete Lagrangian of the form

$$
\mathcal{L}=\mathcal{L}\left(w^{(n)}, w^{(n+1)}, w^{(n+2)}\right),
$$

since the discrete Euler-Lagrange equations

$$
\sum_{i=0}^{2} \frac{\partial}{\partial w^{(n)}} \mathcal{L}\left(w^{(n-i)}, w^{(n+1-i)}, w^{(n+2-i)}\right)=0
$$

do not generate terms containing products $w^{(j)} w^{k)}$ with $|j-k|>2$. In order to obtain a discretization with a Lagrangian structure, we fix $n=0$ and take a discrete Lagrangian of the form

$$
\mathcal{L}\left(w^{(0)}, w^{(1)}, w^{(2)}\right)=\mathcal{T}-\mathcal{V},
$$


where the discrete fourth derivative is generated by the term

$$
\mathcal{T}=\frac{1}{2 h^{4}}\left(2\left(w^{(0)}-w^{(1)}\right)^{2}-\left(w^{(0)}-w^{(2)}\right)^{2}+2\left(w^{(1)}-w^{(2)}\right)^{2}\right),
$$

and the other terms are specified by

$$
\mathcal{V}=\mathcal{V}_{5}+\mathcal{V}_{3}+\frac{c}{3}\left(w^{(0)}+w^{(1)}+w^{(2)}\right)
$$

with

$$
\begin{aligned}
\frac{5}{a} \mathcal{V}_{5}= & \alpha_{0} w^{(0)}\left(w^{(1)}\right)^{3} w^{(2)}+\frac{1}{2} \alpha_{1}\left(\left(w^{(0)}\right)^{2}\left(w^{(1)}\right)^{3}+\left(w^{(1)}\right)^{2}\left(w^{(2)}\right)^{3}\right) \\
& +\frac{1}{2} \alpha_{2}\left(\left(w^{(1)}\right)^{2}\left(w^{(0)}\right)^{3}+\left(w^{(2)}\right)^{2}\left(w^{(1)}\right)^{3}\right) \\
& +\frac{1}{2} \alpha_{3}\left(w^{(0)}\left(w^{(1)}\right)^{4}+w^{(1)}\left(w^{(2)}\right)^{4}\right) \\
& +\frac{1}{2} \alpha_{4}\left(w^{(1)}\left(w^{(0)}\right)^{4}+w^{(2)}\left(w^{(1)}\right)^{4}\right) \\
& +\frac{1}{3} \alpha_{5}\left(\left(w^{(0)}\right)^{5}+\left(w^{(1)}\right)^{5}+\left(w^{(2)}\right)^{5}\right),
\end{aligned}
$$

and

$$
\begin{aligned}
\frac{3}{b} \mathcal{V}_{3}= & \beta_{0} w^{(0)} w^{(1)} w^{(2)}+\frac{1}{2} \beta_{1}\left(w^{(0)}\left(w^{(1)}\right)^{2}+w^{(1)}\left(w^{(2)}\right)^{2}\right) \\
& +\frac{1}{2} \beta_{2}\left(w^{(1)}\left(w^{(0)}\right)^{2}+w^{(2)}\left(w^{(1)}\right)^{2}\right) \\
& +\frac{1}{3} \beta_{3}\left(\left(w^{(0)}\right)^{3}+\left(w^{(1)}\right)^{3}+\left(w^{(2)}\right)^{3}\right),
\end{aligned}
$$

where, in (31) and (32) we have taken affine combinations, so that the coefficients are required to satisfy

$$
\sum_{j=0}^{5} \alpha_{j}=1=\sum_{j=0}^{3} \beta_{j}
$$

in order to ensure the correct continuum limit, and we have included all possible terms of degrees 5 and 3, respectively, except those whose discrete variational derivative produces expressions of degree greater than one in $w^{(-2)}$ or $w^{(2)}$ (we have also grouped together terms having the same variational derivative). Hence we arrive at a discretization of (24) which is explicit and birational, being given by

$$
\frac{w^{(-2)}-4 w^{(-1)}+6 w^{(0)}-4 w^{(1)}+w^{(2)}}{h^{4}}=\hat{F}_{4}+\hat{F}_{2}+c,
$$

where the quartic and quadratic terms are given by

$$
\begin{aligned}
\frac{5}{a} \hat{F}_{4}= & \alpha_{0}\left(w^{(-2)}\left(w^{(-1)}\right)^{3}+3 w^{(-1)}\left(w^{(0)}\right)^{2} w^{(1)}+\left(w^{(1)}\right)^{3} w^{(2)}\right) \\
& +\alpha_{1}\left(3\left(w^{(-1)}\right)^{2}\left(w^{(0)}\right)^{2}+2 w^{(0)}\left(w^{(1)}\right)^{3}\right) \\
& +\alpha_{2}\left(2\left(w^{(-1)}\right)^{3} w^{(0)}+3\left(w^{(0)}\right)^{2}\left(w^{(1)}\right)^{2}\right) \\
& +\alpha_{3}\left(4 w^{(-1)}\left(w^{(0)}\right)^{3}+\left(w^{(1)}\right)^{4}\right)+\alpha_{4}\left(\left(w^{(-1)}\right)^{4}+4\left(w^{(0)}\right)^{3} w^{(1)}\right) \\
& +5 \alpha_{5}\left(w^{(0)}\right)^{4},
\end{aligned}
$$




$$
\begin{aligned}
\frac{3}{b} \hat{F}_{2}= & \beta_{0}\left(w^{(-2)} w^{(-1)}+w^{(-1)} w^{(1)}+w^{(1)} w^{(2)}\right) \\
& +\beta_{1}\left(2 w^{(-1)} w^{(0)}+\left(w^{(1)}\right)^{2}\right) \\
& +\beta_{2}\left(\left(w^{(-1)}\right)^{2}+2 w^{(0)} w^{(1)}\right)+3 \beta_{3}\left(w^{(0)}\right)^{2},
\end{aligned}
$$

respectively. A general approach to Lagrangian fourth-order difference equations and their continuum limits appears in the recent paper [9].

An advantage of using the Lagrangian discretization (33) is that it is symplectic; so it is a birational symplectic integrator. This can be seen from the discrete analogue of the Ostrogradsky transformation, introduced in [2], which provides canonical variables $q_{1}, p_{1}, q_{2}, p_{2}$ via the formulae

$$
\begin{aligned}
& q_{1}=w^{(0)}, \quad p_{1}=\mathcal{L}_{1}\left(w^{(-1)}, w^{(0)}, w^{(1)}\right)+\mathcal{L}_{2}\left(w^{(-2)}, w^{(1)}, w^{(0)}\right), \\
& q_{2}=w^{(1)}, \quad p_{2}=\mathcal{L}_{2}\left(w^{(-1)}, w^{(0)}, w^{(1)}\right),
\end{aligned}
$$

where

$$
\mathcal{L}_{j}=\frac{\partial \mathcal{L}}{\partial w^{(j)}}\left(w^{(0)}, w^{(1)}, w^{(2)}\right), \quad j=0,1,2 .
$$

In terms of these variables, the four-dimensional map defined by (33) preserves the canonical symplectic form

$$
\omega=\mathrm{d} p_{1} \wedge \mathrm{d} q_{1}+\mathrm{d} p_{2} \wedge \mathrm{d} q_{2},
$$

and this immediately implies that it preserves the volume form $\omega \wedge \omega$, so it is measure-preserving.

Qualitatively it appears that the approximate solutions of (24) provided by these two discretizations are somewhat similar. To see this, one can consider solutions in the neighbourhood of a fixed point. If $a b \neq 0$ then, by scaling $w$ and $x$, the parameters can be taken as

$$
a=1, \quad b=-2 \epsilon, \quad c=1-\delta,
$$

with $\epsilon^{2}=1$ and $\delta$ arbitrary. Then (24) has fixed points at $w= \pm \sqrt{\epsilon \pm \sqrt{\delta}}$, so that $\delta \geq 0$ is a necessary condition for reality, and then generically there are either four, two or zero real fixed points depending on the choice of $\epsilon= \pm 1$ and the value of $\delta$. In particular, let us take the case

$$
\epsilon=1, \quad 0<\delta<1
$$

when there are four real fixed points, one of which is at $w=w^{*}$, where

$$
w^{*}=\sqrt{1+\sqrt{\delta}}
$$

The eigenvalues of the linearization of (24) around this point consist of a real pair $\pm \gamma$ and an imaginary pair $\pm \mathrm{i} \gamma$, for $\gamma=\left(4 w^{*} \sqrt{\delta}\right)^{1 / 4}$, corresponding to one stable direction, one unstable direction, and a two-dimensional centre manifold. The discretizations (26) and (33) both have the same fixed points as the original 
differential equation, and using the fact that (26) is reversible, and that (33) is symplectic (and also reversible), together with standard facts about linear stability of reversible/symplectic maps (see [13] or [17]), in each case the characteristic polynomial of the linearization around a fixed point is palindromic (equivalently, $\lambda$ is a root if and only if $\lambda^{-1}$ is). If we consider the linearization around $w^{*}$, then in both cases we find two real eigenvalues that are reciprocals of one another, corresponding to the stable and unstable directions, together with a complex conjugate pair of eigenvalues of modulus one, giving a two-dimensional centre manifold, just as for the differential equation; and similar considerations apply to the other fixed points. Thus, to a first approximation, the qualitative behaviour of the two discretizations is the same.

\section{Conclusions}

We have found that the higher order analogue of Kahan's method proposed here preserves integrability in the second order example of the quartic oscillator (13) that we have considered, while in the case of a nonlinear beam equation of fourth order the resulting discretization (26) is measure-preserving, and its qualitative behaviour looks similar to that of the Lagrangian discretization (33). In future work we would like to apply this discretization method to other ODE systems of higher order, as well as looking for first integrals of the particular fourth order maps (26) and (33) using the method of discrete Darboux polynomials as described in [5] and [6].

\section{Acknowledgments}

ANWH is supported by Fellowship EP/M004333/1 from the Engineering \& Physical Sciences Research Council, UK, and he thanks the School of Mathematics and Statistics, University of New South Wales, for hosting him as a Visiting Professorial Fellow with funding from the Distinguished Researcher Visitor Scheme. He is also grateful to John Roberts and Wolfgang Schief for providing additional support during his stay in Sydney, where the idea behind this work originated, and to his colleagues at La Trobe for hospitality during his visit to Melbourne in May 2019. Part of this work was carried out at the Isaac Newton Institute, during the GCS programme, supported by EPSRC grant EP/R014604/1. GRWQ is grateful to the Simons Foundation for a grant supporting this work.

\section{References}

1. M. Błaszak, Multi-Hamiltonian Theory of Dynamical Systems, Springer, 1998.

2. M. Bruschi, O. Ragnisco, P. M. Santini and G-Z. Tu, Integrable symplectic maps, Physica D 49 (1991) 273-294.

3. E. Celledoni, R.I. McLachlan, B. Owren and G.R.W. Quispel, Geometric properties of Kahan's method, J. Phys. A 46 (2013) 025201. 
4. E. Celledoni, R.I. McLachlan, D.I. McLaren, B. Owren and G.R.W. Quispel, Discretization of polynomial vector fields by polarization, Proc. R. Soc. A 471 (2015) 20150390.

5. E. Celledoni, C.Evripidou, D.I. McLaren, B.Owren, G.R.W.Quispel, B.K. Tapley and P. van der Kamp, Using discrete Darboux polynomials to detect and determine preserved measures and integrals of rational maps, J. Phys. A 52 (2019) 31LT01.

6. E. Celledoni, C.Evripidou, D.I. McLaren, B.Owren, G.R.W.Quispel and B.K. Tapley, Detecting and determining preserved measures and integrals of rational maps, in preparation.

7. L. Da Vinci, Codex Madrid, vol. I, 1493.

8. O. Esen, M. de León, C. Sardón, A Hamilton-Jacobi formalism for higher order implicit systems, arXiv:1901.1030

9. G. Gubbiotti, Lagrangians and integrability for additive fourth-order difference equations, arXiv: 1910.11458

10. S.M. Han, H. Benaroya and T. Wei, Dynamics of Transversely Vibrating Beams using four Engineering Theories, J. Sound Vib. 225 (1999) 935-988.

11. R. Hirota and K. Kimura, Discretization of the Euler top, J. Phys. Soc. Jap. 69 (2000) 627-630.

12. A.N.W. Hone and M. Petrera, Three-dimensional discrete systems of HirotaKimura type and deformed Lie-Poisson algebras, J. Geom. Mech. 1 (2009) 55-85.

13. J.E. Howard and R.S. Mackay, Linear stability of symplectic maps, J. Math. Phys. 28 (1987) 1036-1051.

14. A. Iatrou and J.A.G. Roberts, Integrable mappings of the plane preserving biquadratic invariant curves. J. Phys. A: Math. Gen. 34 (2001) 6617-36.

15. W. Kahan, Unconventional numerical methods for trajectory calculations, unpublished lecture notes, 1993.

16. W. Kahan and R.-C. Li, Unconventional schemes for a class of ordinary differential equations - with applications to the Korteweg-de Vries equation, J. Comp. Phys. 134 (1997) 316-331.

17. A. Lahiri, A. Bhowal and T.K. Roy, Fourth order resonant collisions of multipliers in reversible maps: Period-4 orbits and invariant curves, Physica D 85 (1995) 10-24.

18. R.E. Mickens, Nonstandard finite difference models of differential equations, World Scientific, 1994.

19. R.E. Mickens, A nonstandard finite-difference scheme for the Lotka-Volterra system, Appl. Num. Math. 45 (2003) 309-314.

20. M. Petrera, A. Pfadler and Yu.B. Suris, On integrability of Hirota-Kimura type discretizations, Regular Chaotic Dyn. 16 (2011) 245-89.

21. M. Petrera and R. Zander, New classes of quadratic vector fields admitting integralpreserving Kahan-Hirota-Kimura discretizations, J. Phys. A: Math. Theor. 50 (2017) 205203.

22. M. Petrera, J. Smirin and Yu.B. Suris, Geometry of the Kahan discretizations of planar quadratic Hamiltonian systems, Proc. R. Soc. A 475 (2019) 20180761.

23. R.B. Potts, Best difference equation approximation to Duffing's equation, J. Austral. Math. Soc. Ser. B 23 (1982) 349-356.

24. R.B. Potts, Weierstrass elliptic difference equations, J. Austral. Math. Soc. Ser. B 35 (1987) 43-48.

25. G.R.W. Quispel, J.A.G. Roberts and C.J. Thompson, Integrable mappings and soliton equations, Phys Lett A 126 (1988) 419-421.

26. J.M. Sanz-Serna, An unconventional symplectic integrator of W. Kahan, Appl. Num. Math. 16 (1994) 245-250. 
27. Y. Shi and J. Xu, Quasi-periodic solutions for a class of coupled beam equation system, Discrete Cont. Dyn. B 25 (2020) 31-53. 
27. Y. Shi and J. Xu, Quasi-periodic solutions for a class of coupled beam equation system, Discrete Cont. Dyn. B 25 (2020) 31-53. 